\title{
Theoretical and experimental analysis of current spreading in AIGalnP light emitting diodes
}

\author{
R. M. Perks, ${ }^{\text {a) }}$ A. Porch, and D. V. Morgan \\ Cardiff School of Engineering, Queens Buildings, The Parade, Cardiff CF24 3AA, United Kingdom \\ J. Kettle \\ The Multidisciplinary Nanotechnology Centre, Swansea University, Singleton Park, Swansea SA2 8PP, \\ United Kingdom
}

(Received 7 April 2006; accepted 21 June 2006; published online 24 October 2006)

\begin{abstract}
The profile of light emission from aluminum gallium indium phosphide (AlGaInP) light emitting diodes has been studied and compared with the theoretical modeling of current spreading using a lossy transmission line model of current injected into the active region. Discrepancies between the experimentally determined emission profile and the theoretically predicted current injected into the active region have been analyzed and explained using a Monte Carlo ray tracing simulation which considers the trajectories of the photons after leaving the active region and the particular geometry of the device current, die size, and current spreading layer resistance upon the emitted emission profile. The combination of the current spreading and ray tracing models give very good agreement with the experimental emission profiles for both thin and thick current spreading layers. (C) 2006 American Institute of Physics. [DOI: 10.1063/1.2358396]
\end{abstract}

\section{INTRODUCTION}

Aluminum gallium indium phosphide (AlGaInP) alloys were developed in the 1980s, and today AlGaInP is the primary material system for high-brightness light emitting diodes (LEDs) in the long wavelength part of visible spectrum. ${ }^{1,2}$ A typical LED structure consists of an active region sandwiched between two carrier-confining layers, thus forming a double-heterojunction structure. ${ }^{3-5}$ Issues relating to external quantum efficiency arise in such devices since current is injected via an opaque metal contact, so that light generated in the active region below the contact is shadowed by the contact itself. This problem is further exacerbated by the low conductivity of the top $p$-type cladding layer of the LED (Refs. 2-5) owing to the $p$-type doping density saturating at relatively low levels (around 5 $\times 10^{17} \mathrm{~cm}^{-3}$ ), which results in the inefficient lateral spreading of the injected current. A current spreading layer fabricated from a transparent conductor of low sheet resistance is therefore employed in all high efficiency devices in order to spread current throughout the p-cladding region of the LED and increase the current-injected area without having to increase the size or geometry of the contact. Practical current spreading layers composed of $\mathrm{AlGaAs},{ }^{6} \mathrm{GaP}^{7}$ and indium tin oxide $^{8}$ (ITO) have been realized.

Morgan et al. ${ }^{4}$ described measurements of an exponential decay in light emission intensity radially outwards to the perimeter of the device, with the rate of decay increasing with increasing sheet resistance. A simple, infinite transmission line model was developed to characterize current spreading layers such as those grown onto $\mathrm{AlGaInP}$ surface emitting LEDs. Assuming that the injected current is proportional to the emission intensity, good agreement between this

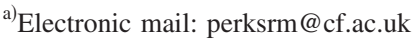

model and the experimental data for ITO material was obtained. ${ }^{4}$ This analysis has been extended so that the current spreading through current spreading layers of finite radial size can be calculated ${ }^{9}$ and will be developed in Sec. II (below) in order to carry out a detailed comparison with recent experimental work.

\section{ANALYTICAL MODELING OF CURRENT SPREADING IN LEDS}

\section{A. Finite transmission line model for circular geometry devices}

A lossy transmission line model proposed by Morgan et $a l^{4}$ for calculating current spreading has been quantified by Morgan and Porch ${ }^{9}$ for circular device geometries. The analysis can be extended to account for finite die size, as will be shown below, from which the radial dependence of both the radial spreading current density $J_{s}(r)$ and the current density $J_{i}(r)$ injected into the $p n$ junction outside of the contact (when $r>r_{0}$ ) can be calculated. An annular unit cell of the transmission line is shown in Fig. 1, of width $\Delta r$ and positioned at some radial distance $r$ from the center of the metal contact.

Defining $\rho$ and $R_{\square}=\rho / t$ to be the resistivity and sheet resistance, respectively, of a spreading layer of thickness $t$ and $G=d J_{i} / d V$ to be the conductance per unit area of the $p n$ junction underneath it, the series resistance and shunt conductance of the unit cell are $\Delta R=R_{\square} \Delta r / 2 \pi r$ and $\Delta G$ $=2 \pi r G \Delta r$, respectively. For simplicity, it is assumed that $G$ is independent of bias voltage above the turn-on voltage $V_{0}$ of the junction; then the injected current density is $J_{i}=0$ for $V \leqslant V_{0}$ and $J_{i}=G\left(V-V_{0}\right)$ for $V>V_{0}$. The equivalent circuit of the unit cell and the idealized junction characteristic are both shown in Fig. 2. When current is injected into the LED it is assumed that the metal contact forms an equipotential sur- 

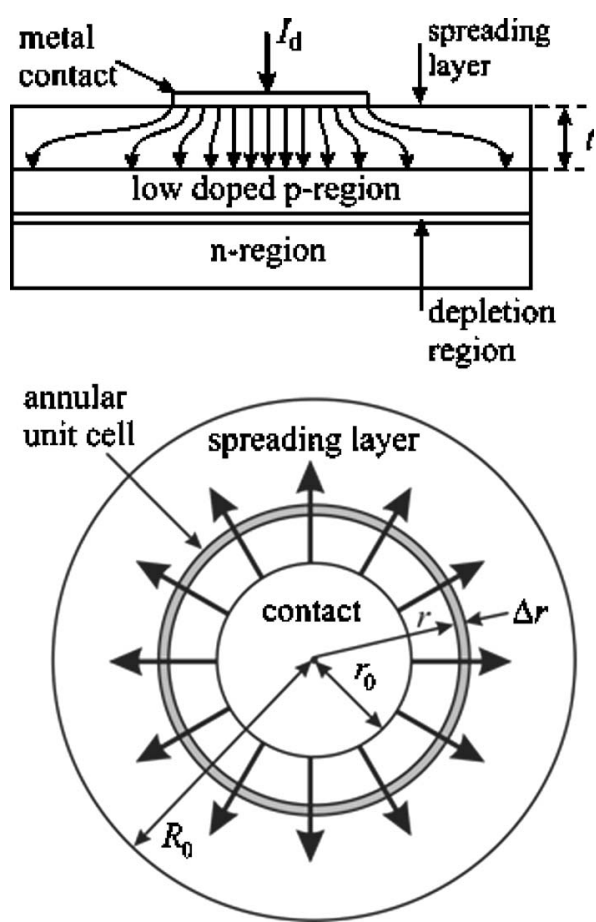

FIG. 1. Schematic cross section and plan views of a typical surface emitting LED of circular geometry.

face at potential $V\left(r_{0}\right)>V_{0}$, which sets the bias point $Q$ for the $p n$ junction beneath the contact (i.e., in the region $r$ $\left.\leqslant r_{0}\right)$; note that there will be no potential difference across the spreading layer if it is very thin, as is the case here. The spreading layer potential $V(r)$ then decreases with increasing radial distance $r\left(>r_{0}\right)$ away from the edge of the contact, so that the bias point slides down the characteristic towards the turn-on point. Within this simple model it can be seen that a potential $V_{0}$ is attained only at the perimeter of a spreading layer of infinite extent; for a finite spreading layer, the perimeter potential is always at some value above $V_{0}$ [but below $\left.V\left(r_{0}\right)\right]$.

The analysis of the circular unit cell using simple circuit theory yields, for the potential $V(r)$ at radial distances $r>r_{0}$,

$$
\frac{d}{d r}\left(r \frac{d V}{d r}\right)-R_{\square} G r\left(V-V_{0}\right)=0,
$$

which has solutions $V(r)=V_{0}+A K_{0}(\alpha r)+B I_{0}(\alpha r)$, where $I_{0}(x)$ and $K_{0}(x)$ are zeroth order, modified Bessel functions of the first and second kinds, respectively, $A$ and $B$ are con-

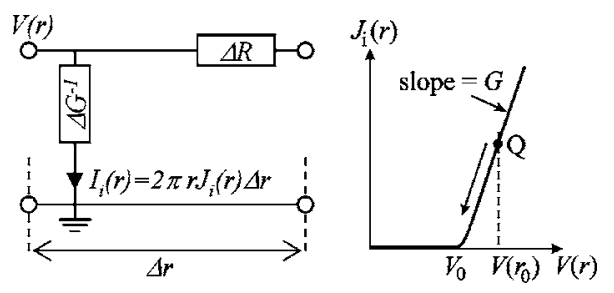

FIG. 2. Equivalent circuit used for calculating the radial dependence of the current spreading where $\Delta R=R_{\square} \Delta r / 2 \pi r$ and $\Delta G=2 \pi r G \Delta r$. Also shown is the idealized characteristic for the current density injected into the $p n$ junction assumed for the transmission line model.

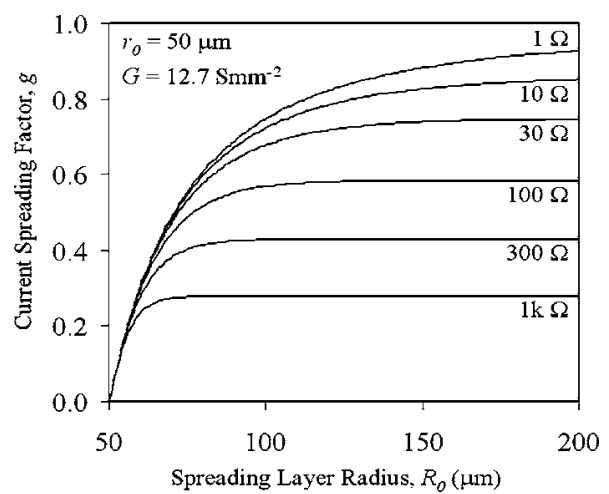

FIG. 3. The current spreading factor $g$ as a function of spreading layer radius $R_{0}$ for various values of spreading layer sheet resistance $R_{\square}$. In terms of the contact radius $r_{0}, g$ reaches a maximum limiting value (i.e., $g_{\max }$ ) provided that $\alpha\left(R_{0}-r_{0}\right)>1$.

stants, and $\alpha=\sqrt{R_{\square} G}$. The parameter $\ell_{D}=1 / \alpha$ sets the length scale (i.e., the decay length) of the radial fall off of both the spreading current density $J_{s}(r)$ and injected current density $J_{i}(r)$ away from the contact edge. In terms of $V(r)$, the spreading current density is then

$$
J_{s}(r)=-\frac{1}{R_{\square} t} \frac{d V}{d r}=\frac{1}{t} \sqrt{\frac{G}{R_{\square}}}\left[B K_{1}(\alpha r)-A I_{1}(\alpha r)\right],
$$

where $I_{1}(x)$ and $K_{1}(x)$ are first order, modified Bessel functions of the first and second kinds, respectively. There is an open circuit boundary condition at the perimeter of the spreading layer (defined to have radius $R_{0}$ ) so that $J_{s}\left(R_{0}\right)$ $=0$ and $B=A I_{1}\left(\alpha R_{0}\right) / K_{1}\left(\alpha R_{0}\right)$.

Light is emitted from the device as the spreading current is injected into the active region of the $p n$ junction at radial distances $r_{0}<r \leqslant R_{0}$ (i.e., outside the region shadowed by the contact). From the unit cell of Fig. 2, the associated injected current density is $J_{i}(r)=G\left(V(r)-V_{0}\right)$ and therefore,

$$
J_{i}(r)=\alpha t J_{s}\left(r_{0}\right)\left[\frac{I_{0}(\alpha r) K_{1}\left(\alpha R_{0}\right)+I_{1}\left(\alpha R_{0}\right) K_{0}(\alpha r)}{I_{1}\left(\alpha R_{0}\right) K_{1}\left(\alpha r_{0}\right)-I_{1}\left(\alpha r_{0}\right) K_{1}\left(\alpha R_{0}\right)}\right] \text {. }
$$

Calculations of the spreading current density as a function of sheet resistance $R_{\square}$ and spreading layer radius $R_{0}$ are shown in Figs. 3 and 4, assuming that $G=1.27 \mathrm{~S} / \mathrm{mm}^{2}$ for a fixed contact radius of $r_{0}=50 \mu \mathrm{m}$ (both in accordance with published experimental data $\left.{ }^{1-3,6}\right)$. Figure 3 shows the frac-

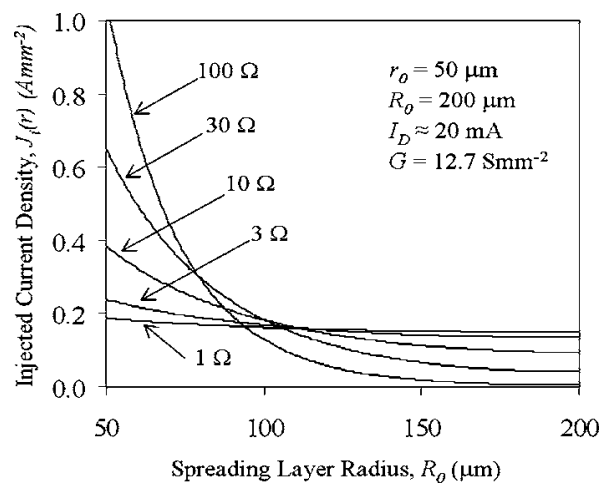

FIG. 4. The injected current density $J_{i}(r)$ (proportional to the radial dependence of the optical output power) as a function of radial distance $r$ from the center of the contact for various values of spreading layer resistance $R_{\square}$. 


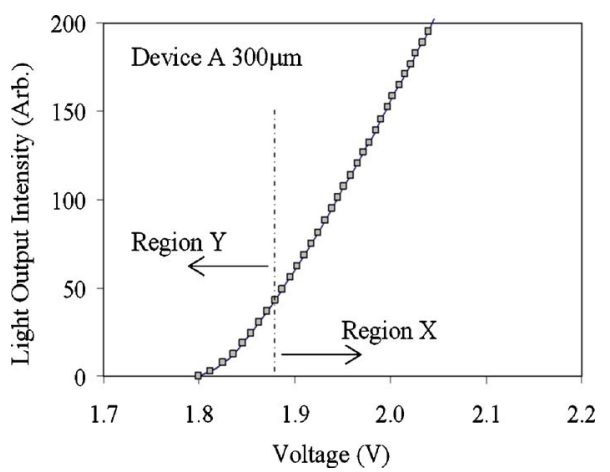

FIG. 5. Typical light-voltage $(L-V)$ characteristic showing LED turn on voltage.

tion of total current that is spread (denoted by the dimensionless current spreading factor $g$ ) as a function of $R_{0}$ for a range of different spreading layer sheet resistances, while Fig. 4 shows the radial dependence of the injected current density $J_{i}(r)$ for a fixed value of $R_{0}=200 \mu \mathrm{m}$, again for various sheet resistances. These results exhibit the anticipated trends; a high sheet resistance sets a small value of decay length $\ell_{D}$ (typically $\ll R_{0}-r_{0}$ ), resulting in an approximately exponential falloff of $J_{i}(r)$ and a small fraction of total current spread laterally; a low sheet resistance sets a large value of $\ell_{D}$ (which can be greater than $R_{0}-r_{0}$ ), thus spreading almost all of the total current (for the ratios of $R_{0} / r_{0}>3$ or more, as met in practice), and results in an approximately uniform radial dependence of $J_{i}(r)$. Considering some specific numerical examples from Fig. 4, a spreading layer of sheet resistance $R_{\square}=1000 \Omega$ gives a decay length of $\ell_{D}$ $\approx 28 \mu \mathrm{m}$, while $R_{\square}=100 \Omega$ gives $\ell_{D} \approx 89 \mu \mathrm{m}$; these values of $\ell_{D}$ correspond closely with the values of $R^{\prime} \equiv R_{0}-r_{0}$ beyond which $J_{i}(r)$ becomes approximately constant. However, when $R_{\square}=10 \Omega$ it is found that $\ell_{D} \approx 280 \mu \mathrm{m}$, so that the limit $R^{\prime}>\ell_{D}$ is not attained unless the spreading layer radius is increased beyond a radius $R_{0} \approx 330 \mu \mathrm{m}$ (i.e., a large die size).

The radial falloff of the injected current density [as defined by Eq. (3)] is difficult to measure in a conventional semiconductor structure. However, the measurement of the radial dependence of the emitted light of an LED can be used to infer the current distribution and hence experimentally verify the predictions of Eq. (3). To do this it is necessary to understand the relationship between the emitted light intensity $L(r)$ as a function of the local depletion region potential across the device $V(r)$. This has been done for the devices reported here. Figure 5 shows a typical result of the data obtained by Morgan et al. ${ }^{4}$ The conclusion drawn from this data is that light ceases to be emitted when the potential falls below a critical experimental value $V_{c}$ that is close to the turn-on voltage $V_{0}$ of the idealized diode characteristic assumed in the modeling. Above this voltage, to a very good approximation the light intensity is $L(r) \propto V(r)-V_{c}$, in accordance with the data of Fig. 5.

An important limitation of modeled data shown in Fig. 4, when it is used to compute the current distribution in the active region of an LED, is that it does not account for geometrical effects associated with the thickness of the current spreading layer when considering the light output from a device. One such example is the shadowing of the emitted light in the vicinity of the metal contact, which could reduce the intensity of light output from the contact vicinity or alternatively underestimate the flux of photons emerging by multiple scattering routes. To overcome these limitations, complementary modeling has been carried out using a Monte Carlo ray tracing simulation method.

\section{B. Monte Carlo ray tracing studies}

A Monte Carlo ray tracing simulation that models the light propagation through a current spreading layer has been developed. This type of simulation is useful for modeling LEDs since the light emission from an LED is inherently incoherent and spontaneous. Previous simulations have taken a similar approach, ${ }^{10,11}$ but our method is superior as it also models the current distribution in the $p n$ junction of AlGaInP LEDs derived from the transmission line model. This allows the accurate simulation of light profiles for devices with different material properties and current spreading layer thicknesses, thus allowing more accurate comparisons to be made with the experimental results. In this one dimensional simulation, incoherent photons are generated in the active region at random positions and at random angles with respect to the plane of the active region; the photons are being considered as particles and not electromagnetic waves. The simulation follows the trajectory of a single photon through the device, taking into account the main loss mechanisms of absorption, scattering, and interaction with the semiconductor-air interface. This is repeated $10^{8}$ times to give a robust performance indicator of the device.

\section{EXPERIMENTAL RESULTS AND COMPARISON WITH THEORY}

Previous work in this laboratory reported results of experimental profiles obtained by the analysis of photographic plates of the LED emission. ${ }^{4}$ An analysis of this kind has inherent limitations due to the nonlinearity in the sensitivity of the photographic emulsions to light intensity, particularly the saturation of the emulsion at high light intensity levels (please refer to the appendix for an appraisal of this problem). To overcome this, an alternative technique for obtaining the intensity profile data has been used.

The radial distribution of light output intensity $L(r)$ from the LED surfaces were obtained using a $5 \times 10^{6}$ pixel charge coupled device (CCD) camera mounted onto a microscope equipped with a $100 \times$ magnification lens to allow for high resolution imaging. The camera is externally triggered to capture an image at a constant switch-on interval. The images obtained are then converted to an 8 bit grey scale bitmap enabling a light intensity map of the surface of the die to be made. A single line scan is then taken from the center bond pad to the edge of the die, providing a cross section of the radial light intensity as a function of distance from the bond pad. The position of the line scan across each die is constant for each device, allowing a quantitative comparison of $L(r)$ at different diode currents. These line scans are correlated to a scanning electron microscope image of the die 


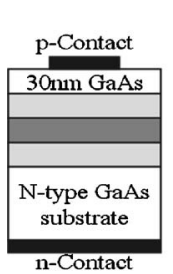

Device A

\begin{tabular}{c|c|c|c}
\hline CSL material & $\rho\left(\Omega \mathrm{m}^{-1}\right)$ & $t(\mu \mathrm{m})$ & Approx. $R_{\square}(\Omega)$ \\
\hline $\begin{array}{c}20 \mathrm{~mm} \text { GaAs } \\
(\text { Device A) }\end{array}$ & $2 \times 10^{-5}$ & 0.2 & 1000 \\
\hline $\begin{array}{c}1 \mu \mathrm{m} \mathrm{GaP} \\
(\text { Device B) }\end{array}$ & $5 \times 10^{-5}$ & 1.0 & 50 \\
\hline $\begin{array}{l}5 \mu \mathrm{m} \mathrm{GaP} \\
\text { (Device C) }\end{array}$ & $5 \times 10^{-5}$ & 5.0 & 10 \\
\hline
\end{tabular}

FIG. 6. Schematic cross-sectional view of the devices used and (inset table) corresponding material properties, showing (A) device with no effective current spreading layer (CSL), (B) a $1 \mu \mathrm{m} \mathrm{GaP} \mathrm{CSL,} \mathrm{and} \mathrm{(C)} \mathrm{a} 5 \mu \mathrm{m} \mathrm{GaP}$ CSL.

surface, thus ensuring that the correct spreading lengths are used in final comparisons with theoretical data.

The devices used for this study were those fabricated for earlier studies ${ }^{3,4}$ with different spreading layers (and thus spreading layer sheet resistances). Schematics of the outline geometries of the three basic devices are shown in Fig. 6 . These devices were fabricated from the same semiconductor layer with an active region consisting of a double heterostructure. ${ }^{4}$ The active region of each consisted of a $1 \mu \mathrm{m}$ thick Zn-doped AlGaInP $p$ layer, with a $0.6 \mu \mathrm{m}$ undoped AlGaInP intrinsic layer and a $1 \mu \mathrm{m}$ Si-doped $n$ layer. Three different current spreading layers were subsequently grown, thus allowing the investigation of the effects of different sheet resistances. Likewise, three square die sizes were cleaved from each wafer $(300,500$, and $700 \mu \mathrm{m})$, corresponding to values of $R^{\prime} \equiv R_{0}-r_{0}$ of 100,200 , and $300 \mu \mathrm{m}$, respectively (here the contact radius is $r_{0}=50 \mu \mathrm{m}$ for all three device geometries). Thus, nine different devices were studied, i.e., three device geometries, each with three different spreading layers. Intensity profiles were obtained in the series-recombination part (i.e., the linear portion) of the diode characteristic, as has been assumed in the derivation of Eq. (3), with a diode conductance per unit area $G$ that is independent of bias voltage at voltages greater than the turn-on voltage $V_{0}$.

Note that the effect of finite die size is being tested here by allowing the decay length $\ell_{D}$ to vary according to the conditions $\ell_{D}<R \prime$ and $\ell_{D}>R^{\prime}$. This can be achieved by varying either the die size $R_{0}$ for a fixed sheet resistance $R_{\square}$ or alternatively by varying $R_{\square}$ for a suitably selected die size. The diodes fabricated for this work present consistent data for both of these alternative approaches.

\section{A. Effect of drive current on emission profiles}

The linearity of the detector system can be tested by measuring the emission profiles for a range of diode drive currents. Consistent profile characteristics (i.e., in terms of general profile shape and associated values of $\ell_{D}$ ) provide a measure of linearity of the detector system. This was carried out for each of the nine devices, with the results indicating

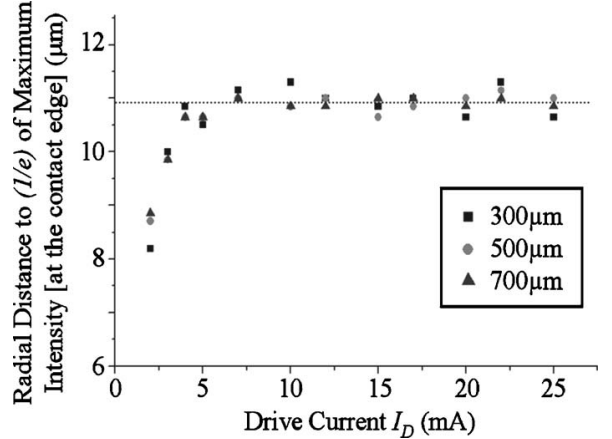

FIG. 7. Radial distance to $(1 / e)$ of the maximum intensity (at the contact edge) as a function of drive current for all three die sizes of device A.

that the functional form of the profiles were unaffected by detector nonlinearities. In the case where $\ell_{D}<R^{\prime}$ (i.e., for high sheet resistance) an exponential radial intensity profile is obtained, in close agreement with the prediction of the transmission line model for an infinite die size. ${ }^{5}$ This is illustrated in Fig. 7, which shows the values of $\ell_{D}$ calculated from light emission profiles obtained for drive currents ranging from 2 to $25 \mathrm{~mA}$. For drive currents between 5 and $25 \mathrm{~mA}, \ell_{D}$ is a constant, indicating that no distortion is observed in the profile shapes and providing evidence that the detector is linear over this range of diode drive currents (up to $25 \mathrm{~mA}$ ).

At lower drive currents $(<5 \mathrm{~mA})$ the decay length $\ell_{D}$ falls off rapidly, the reason for which follows from Fig. 5. The drive current is reduced by decreasing the terminal voltage applied to the diode. At high voltages (such as those corresponding to voltages in region $\mathrm{X}$ in Figure 5), at radial distances $r$ such that $r-r_{0} \geqslant \ell_{D}$ then $V\left(\ell_{D}\right)>V_{c}$, i.e., the diode is still emitting. In these circumstances the optical profile follows closely the spreading current profile. At the lower drive currents, the contact potential is close to the cutoff voltage (e.g., region Y in Fig. 5) and the diode ceases to emit light for values of $r$ such that $r-r_{0}<\ell_{D}$; then there will be a cutoff of intensity, thus giving a reduced (and false) value of $\ell_{D}$, which is in agreement with Fig. 7.

\section{B. Effect of die size on emission profiles}

It has been shown in the previous discussion that the effects of die size become important as the die dimension $R^{\prime}=R_{0}-r_{0}$ approaches the decay length $\ell_{D}$. This problem has been studied experimentally in two ways: by varying the sheet resistance for fixed die size and alternatively by varying the die size itself. The results are summarized in Figs. 8 and 9 for devices of overall die size set at 300, 500, and $700 \mu \mathrm{m}$ (square). Devices labeled A, B, and C have different spreading layer sheet resistances of $R_{\square}=1000,50$, and $10 \Omega$ respectively.

Device A has an ineffective spreading layer, consisting of low doped $p$-type AlGaInP with $30 \mathrm{~nm}$ of doped GaAs cap layer (whose main function is to protect the AlGaInP layer from oxidizing and to ensure a good Ohmic contact). In this case, all of the devices of different die sizes exhibited an exponential falloff of light intensity, with $\ell_{D}$ always less than $R^{\prime}$. Device B has a $1 \mu \mathrm{m}$ thick GaP spreading layer and also 

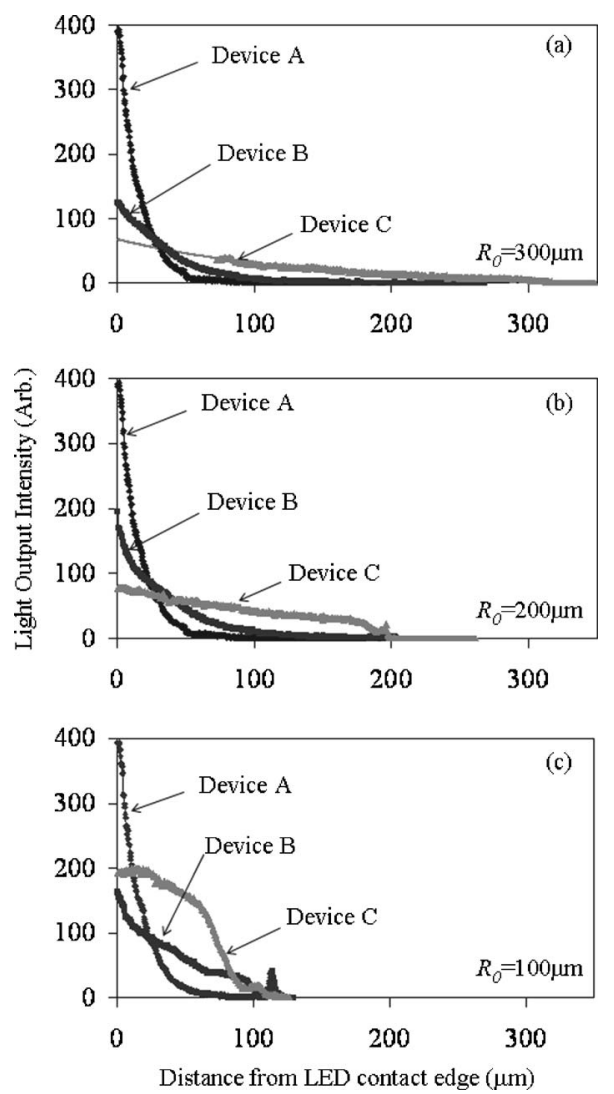

FIG. 8. Comparison of the light emission profiles for different CSLs. (a) $R_{0}=300 \mu \mathrm{m}$, (b) $R_{0}=200 \mu \mathrm{m}$, and (c) $R_{0}=100 \mu \mathrm{m}$. $I_{d} \approx 15 \mathrm{~mA}$ in each case.

shows an exponential falloff at large die sizes. However, for the $300 \mu \mathrm{m}$ die size, the light intensity $L(r)$ does not drop to zero at the edge of the die, unlike device A; additionally, a shoulder appears in the emission profile at the device periphery, consistent with Kish and Fletcher ${ }^{7}$ [Fig. 8(b)] and thus represents a departure from the exponential form, as predicted by theory (see Fig. 4).

Device $\mathrm{C}$ has a very effective $5 \mu \mathrm{m}$ thick GaP current spreading layer, whose emission profile exhibits the most significant departure from exponential [Fig. 8(c)]. Two features are noted. Firstly, the shoulder near the periphery of the die rises, almost to the point of uniform light emission across the die. Secondly, the intensity around the metal contact has increased beyond that of device B. The sequence of emission profiles, summarized in Figs. 8(a)-8(c), provides significant insight on the nature of the transition from an infinite to a finite die size. [Note: The region up to $70 \mu \mathrm{m}$ from the contact for device $\mathrm{C}$ in Fig. 8(a) was shadowed by a large wire bond; the emission profile of this region has been extrapolated from the remaining data.]

A peak in the emission profiles at the edge of some devices is attributed to light being guided along the active region outwards towards the periphery where, as a result of uneven cleaving, it is then directed towards the imaging system. The peak wavelength of this guided light appears to be redshifted in comparison with the surface emission, suggesting that the shorter wavelengths have been reabsorbed in the active region leaving only the "subband" wavelengths to emerge at the far end.
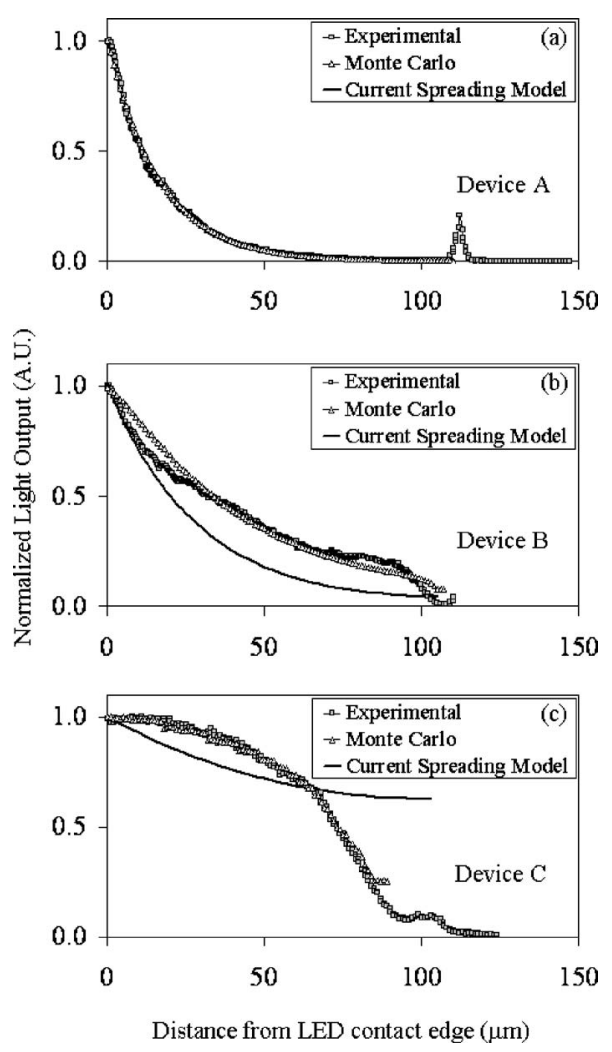

FIG. 9. Normalized comparison of experimental/transmission line data with the Monte Carlo simulation for different CSLs for $R_{0}=300 \mu \mathrm{m}$ and $I_{D}$ $=20 \mathrm{~mA}$.

\section{Comparison with theory}

The data shown in Fig. 8(c) has been replotted along with theoretical curves in Fig. 9. The data shown corresponds to the smallest die size of $300 \mu \mathrm{m}^{2}$, where the edge effect is most clearly observed. In the case of device A (no effective current spreading layer), the experimental profile fits closely to the two theoretical curves, which combine the transmission line model with the Monte Carlo simulation. The presence of the die edge has no effect upon the profile as $R^{\prime} \geqslant 3 \ell_{D}$ in all cases [see Fig. 9(a)].

Figure 9(b) corresponds to the case where the current spreading layer is of intermediate sheet resistance (i.e., $1 \mu \mathrm{m}$ $\mathrm{GaP}$ ), in which case $R^{\prime} \approx \ell_{D}$; consequently, the presence of the die edge influences the emission profile from the die edge inwards. Near the metal contact region the transmission line model fits the experimental data closely but underestimates the emission profile near the die edge. The opposite is true when the Monte Carlo ray tracing is included, agreeing well with the experimental data near the die edge but overestimating the emission profile near the contact. Subtle differences such as these can be attributed, at least in part, to the way that the light intensity has been normalized to the value at the contact edge as well as the possibility of localized diode saturation. Note also that the Monte Carlo technique overestimates the shadowing effect of the metal contact, which only becomes important in the case of the thick spreading layer.

For device $\mathrm{C}$, with a spreading layer of the lowest sheet resistance (i.e., $5 \mu \mathrm{m} \mathrm{GaP}$ ), the presence of the die edge affects the whole emission profile, as shown in Fig. 9(c). In 


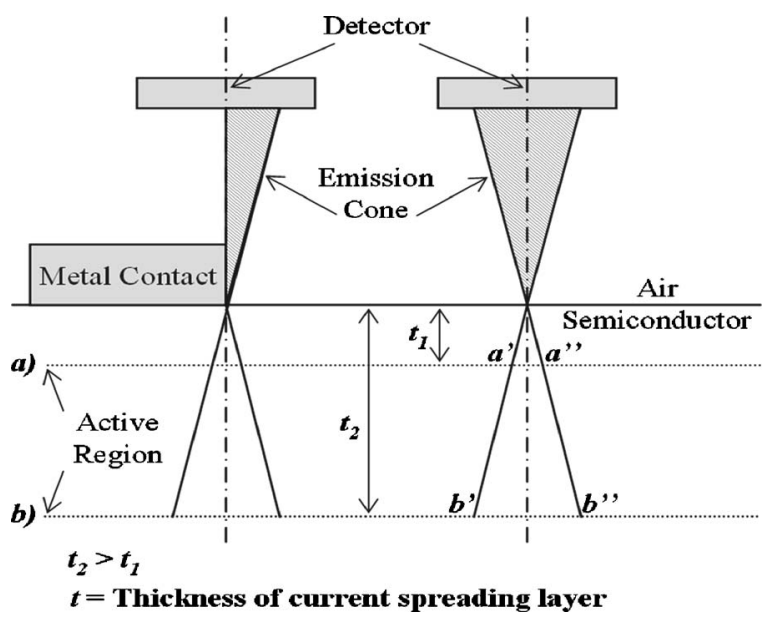

FIG. 10. Schematic illustrating the effect of current spreading layer thickness.

these circumstances, the transmission line model alone yields no quantitative agreement whereas the inclusion of the Monte Carlo ray tracing model shows excellent agreement over the whole emission profile. Thus, geometrical effects govern the emission profile for this device. Qualitatively, this can be understood with the aid of Fig. 10, which compares the light emerging from the surface from a thin current spreading layer (of lower surface located at plane $a$ in Fig. 10) compared with that emerging from a thick one (of lower surface located at plane $b$ ). For any point on the top surface of the LED's spreading layer, the corresponding area in the active region that can couple light to the surface within the critical angle (approximately $19^{\circ}$ for AlGaInP) is larger for thick spreading layers than for thin ones (i.e., the zone in the active region between points $a^{\prime}$ and $a^{\prime \prime}$ is smaller than that between $b^{\prime}$ and $\left.b^{\prime \prime}\right)$. On the other hand, in the vicinity of the metal contact there will be a shadowing effect which will reduce slightly the emitted light. This would account for the decreasing slope in the emission profile in the vicinity of the contact, as seen in Fig. 9(c).

\section{CONCLUSIONS}

This paper presents detailed measurements and analysis of the spatial emission profiles of light emitted from AlGaInP LEDs. The measurements have concentrated on current spreading structures that cover circumstances where the diode current spreading distance $R^{\prime}=R_{0}-r_{0}$ is much larger then the decay length $\ell_{D}$ of the emission profile (an "infinite" die size) and those where $R^{\prime} \ll \ell_{D}$, where the die edges play a major role in the resulting emission profiles. In order to understand and model the transition between these two limiting cases, the infinite transmission line model for current spreading has been extended to incorporate devices of finite die size. The effects of multiple photon scattering and contact shadowing have been included using a ray tracing Monte Carlo simulation and assuming an emitting source of emission profile generated from the transmission line model for current spreading for a device of finite die size.

Particularly important is the fact that the calculated

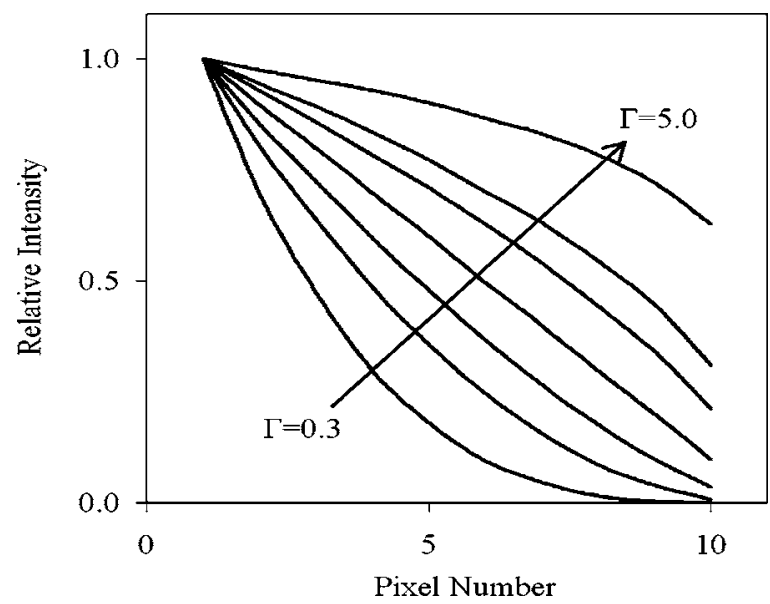

FIG. 11. Synthesized emission profiles with varying gamma correction.

emission profiles for both thin and thick current spreading layers which show good agreement with the experimental data. Referring to the experimental data, it has been established firmly that the detector system is linear for the emission levels considered and that no detector saturation occurs to distort the profiles. This was a serious concern with previous studies ${ }^{4}$ that used photographic emulsion to deduce the emission profiles. We therefore conclude that the extended hybrid Monte Carlo model for emission profiles accounts quantitatively for the detailed structure of the experimental data covering the critical transition from infinite to finite die sizes.

\section{ACKNOWLEDGMENTS}

The authors wish to acknowledge the following: IQE plc of Cypress Drive St. Mellons, Cardiff CF3 OEG, for providing the material used in the fabrication of the devices used in this work, the assistance of Dr. P. Porta at Cardiff School of Physics and Astronomy and Rolfe Wheeler-Jones at Cardiff School of Engineering, and The Multidisciplinary Nanotechnology Centre, Swansea University, Singleton Park, Swansea, SA2 8PP for the Ph.D. studentship for one of the authors (J.K.).

\section{APPENDIX}

The analysis of experimental profiles obtained via the use of photographic plates of LED emission ${ }^{4}$ has inherent limitations due to the nonlinearity in the sensitivity of the photographic emulsions to light intensity. A numerical parameter, gamma, is used to describe the nonlinearity of intensity reproduction. Depending on the characteristics of the emulsion, gamma correction can be employed to restore an image to its original specification. The section below describes how this concept could be attributed to the variation in observed emission profiles.

\section{Gamma correction of synthesized emission profiles}

A "test card" image was generated using an image editing software package on a personal computer. A 10 $\times 11$ pixel, 8 bit gray scale test card bitmap was produced, 
with vertical stripes varying linearly from $100 \%$ white to $100 \%$ black, thus simulating a linearly varying intensity emission profile which was used as a reference. Six further profiles were then generated using the gamma correction feature in the software. This has the effect of changing the optical density response to light of photographic film and is consistent with the concept of gamma correction. Figure 11 shows the variation in synthesized emission profiles for a range of gamma values. For low values of gamma the profile is more exponential. As gamma is increased the profile exhibits shouldering at the perimeter; consistent with the profile observed in Fig. 9(c); this highlights the degree to which emission profiles may vary with data obtained via photographic plates. Clearly, the reliability of photographic data is brought into question when attempting to determine actual LED intensity emission profiles.
${ }^{1}$ K. Streubel, N. Linder, R. Wirth, and A. Jaeger, IEEE J. Sel. Top. Quantum Electron. 8, 321 (2002)

${ }^{2}$ F. A. Kish, D. A. Vanderwater, M. J. Peanaksy, J. G. Yu, R. M. Fletcher, M. Craford, and V. M. Robbins, Appl. Phys. Lett. 64, 2839 (1994).

${ }^{3}$ Y. H. Aliyu, D. V. Morgan, H. Thomas, and S. W. Bland, Electron. Lett. 31, 2210 (1995).

${ }^{4}$ D. V. Morgan, I. M. Al-Ofi, and Y. H. Aliyu, Semicond. Sci. Technol. 15, 67 (2000).

${ }^{5}$ A. Porch, D. V. Morgan, R. M. Perks, M. O. Jones, and P. P. Edwards, J. Appl. Phys. 95, 4737 (2004).

${ }^{6}$ H. Sugawara, K. Itaya, H. Nozaki, and G. Hatakoshi, Appl. Phys. Lett. 61, 1775 (1992).

${ }^{7}$ F. A. Kish and R. M. Fletcher, High Brightness LEDs, edited by M. G. Craford (Academic, New York, 1997), Chap. 5.

${ }^{8}$ D. V. Morgan, Y. H. Aliyu, R. W. Bunce, and A. Salehi, Thin Solid Films 312, 268 (1998).

${ }^{9}$ D. V. Morgan and A. Porch, in Proceedings of the 29th Workshop on Compound Semiconductor Devices and IC's, Cardiff, UK, 2005, p. 5-7.

${ }^{10}$ S. J. Lee, Appl. Opt. 40, 1427 (2001).

${ }^{11}$ S. J. Lee, A. Baldano, and J. Kanicki, IEEE J. Sel. Top. Quantum Electron. 10, 37 (2004). 